\title{
Pathway Analysis of Gene Expression of E14 Versus E18 Fetal Fibroblasts
}

\author{
Michael S. Hu, ${ }^{1,2}$ Mimi R. Borrelli, Michael Januszyk, \\ Anna Luan, Samir Malhotra, Graham G. Walmsley, \\ Wan Xing Hong, ${ }^{1}$ Ruth Tevlin, Geoffrey C. Gurtner, \\ Michael T. Longaker, ${ }^{1,2}$ and Hermann P. Lorenz ${ }^{1, *}$ \\ ${ }^{1}$ Hagey Laboratory for Pediatric Regenerative Medicine, Division of Plastic Surgery, Department of Surgery, Stanford \\ University School of Medicine, Stanford, California. \\ ${ }^{2}$ Institute for Stem Cell Biology and Regenerative Medicine, Stanford University School of Medicine, Stanford, California.
}

Objective: Fetuses early in gestation heal skin wounds without forming scars. The biological mechanisms behind this process are largely unknown. Fibroblasts, however, are cells known to be intimately involved in wound healing and scar formation. We examined fibroblasts in different stages of development to characterize differences in gene expression that may result in the switch from regenerative wound repair to repair with scarring.

Approach: Fibroblasts were isolated and cultured from the back skin of BALB/c wild-type mouse fetuses at embryonic day (E)14 and E18 $(n=10)$. The fibroblast total RNA was extracted, and microarray analysis was conducted using chips containing 42,000 genes. Significance analysis of microarrays was performed to identify genes with greater than twofold expression difference and a false discovery rate of less than two. Identified genes subsequently underwent enrichment analysis to detect differentially expressed pathways.

Results: Two hundred seventy-five genes were differentially expressed between E14 and E18 in fetal fibroblasts. Thirty genes were significantly downregulated and 245 genes were significantly upregulated at E18 compared with E14. Ingenuity pathway analysis identified the top 20 signaling pathways differentially activated in fetal fibroblasts between the E18 and E14 time points.

Innovation: To our knowledge, this work represents the first instance where differentially expressed genes and signaling pathways between fetal fibroblasts at E14 and E18 have been studied.

Conclusion: The genes and pathways identified here potentially underlie the mechanism behind the transition from fetal wound healing via regeneration to wound healing by repair, and may prove to be key targets for future therapeutics.

Keywords: wound healing, scarless repair, regeneration, microarray

\section{INTRODUCTION}

THE PHYSIOLOGICAL RESPONSE to cutaneous injury is a complex and tightly regulated process. Cutaneous injury initiates an acute inflammatory response, followed by a rapid migration of progenitor cells to the wound site. Multipotent cells differentiate into numerous cell types that rapidly form new blood vessels and epithelium. Concurrently, fibroblasts proliferate at the wound site, synthesize collagen, and differentiate into myofibroblasts, which act to contract the wounded skin. Remodeling of the wounded tissue continues far beyond the initial
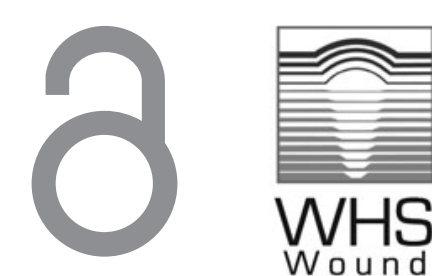

Wound

Healing

Society

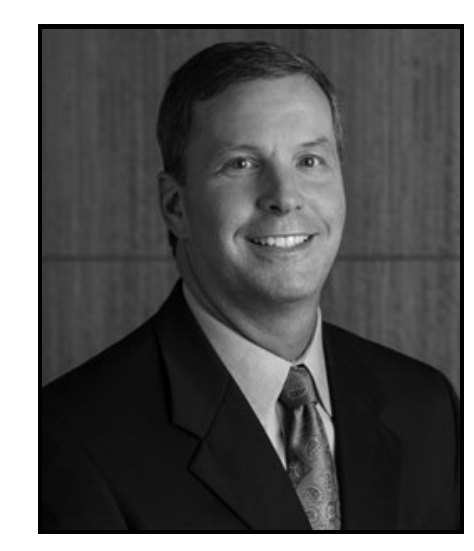

Hermann P. Lorenz, MD

Submitted for publication September 19, 2017. Accepted in revised form September 30, 2017.

*Correspondence: Hagey Laboratory for Pediatric Regenerative Medicine, Division of Plastic Surgery, Department of Surgery, Stanford University School of Medicine, 257 Campus Drive, Stanford, CA 94305-5148

(e-mail: plorenz@stanford.edu). 
injury, and scarring is characterized by the excess accumulation of extracellular matrix (ECM). The organization of the newly synthesized ECM never recapitulates that found in uninjured skin, resulting in a $20-30 \%$ decrease in overall tensile strength in the repaired, compared to normal, skin tissue. ${ }^{1}$

Interestingly, Rowlatt described the ability of the human fetus, early in gestation, to repair cutaneous wounds without forming a scar. ${ }^{2}$ Further work has subsequently confirmed these findings in both animal models and in human fetuses. ${ }^{3}$ The finding of scarless wound healing in human skin suggests there is potential that adult skin can be stimulated to function as fetal skin, and heal via regeneration rather than fibrosis. The mechanisms of scarless healing, however, require further characterization before such knowledge can be applied for therapeutic benefit.

There are numerous differences documented between fetal and adult wound healing phenotypes. Scarless wound healing is age dependent; there is a distinct switch from regenerative to scarring healing around 24 weeks of gestation in human embryos ${ }^{4}$ and around gestational age 18 days (embryonic day [E]18) in mice. ${ }^{5}$ Scar-free healing is also dependent on the size of the wound, with larger wounds healing via scarring at earlier gestational ages. ${ }^{4}$ In vitro, fetal skin fibroblasts are able to simultaneously proliferate and synthesize collagen, whereas adult skin fibroblasts proliferate before they synthesize collagen, suggesting a central role of fibroblasts in the mechanism of scarless wound healing. ${ }^{6}$ This study focused on the difference in fibroblast function at different ages of gestation. A microarray transcriptional profiling comparison was conducted on fetal fibroblasts harvested from mouse fetuses at E14 and E18 to detect any deviations in transcriptomes between scarless and scarring repair. The aim was to identify novel pathways involving fibroblasts that promote regenerative repair and scarless healing.

\section{CLINICAL PROBLEM ADDRESSED}

Scarring is the expected outcome of the human adult wound healing process and is a significant medical issue that can substantially reduce patients' quality of life. Numerous physiological and psychological consequences result from scarring, either the result of trauma or surgery. ${ }^{7}$ Facial scars are often cosmetically displeasing and result in substantial psychosocial distress. Keloid scars can cause immense pain and severe itching, while hypertrophic scars can lead to contracture, erosion of skeletal structure, and potentially lifelong disabil- ity. ${ }^{8}$ Scars and their associated consequences surmount to enormous economic cost estimated in the tens of billions of dollars. ${ }^{9}$

\section{MATERIALS AND METHODS \\ Animals}

BALB/c wild-type mice at 6 weeks of age were purchased from Charles River Laboratories (Wilmington, MA). After acclimation for at least 1 week, male and female mice were bred overnight. Every day, the female mice were checked and the day of vaginal plug was determined to be E0.5 for gestational timing. All animal procedures were performed in accordance with National Institutes of Health (NIH) guidelines according to universityapproved protocols. Mice were closely monitored by the Stanford Administrative Panel on Laboratory Animal Care (APLAC).

\section{Primary cell culture}

Gestational age E14 and E18 pregnant mice were sacrificed with $\mathrm{CO}_{2}$ and cervical dislocation. Fetal mice were surgically removed using sterile technique. Under a dissecting microscope, E14 $(n=10)$ and E18 $(n=10)$ fetal mouse dorsal skin was collected and pooled for fibroblast isolation and primary cell culture under sterile conditions.

Fibroblast primary cell culture was conducted by mincing tissue and treating it with $0.25 \%$ trypsin/ ethylenediaminetetraacetic acid (EDTA) in $37^{\circ} \mathrm{C}$ with mild agitation for $10 \mathrm{~min}$. Mouse embryonic fibroblast culture medium consisting of Dulbecco's modified Eagle's medium, GlutaMAX supplement (Thermo Fisher Scientific, Waltham, MA), 10\% fetal bovine serum (Omega Scientific, Tarzana, CA), $0.1 \mathrm{mM}$ 2-mercaptoethanol (Sigma, St. Louis, MO), and $1 \%$ penicillin/streptomycin (Thermo Fisher Scientific) was used for cell culture. Cells were cultured at $37^{\circ} \mathrm{C}$ in a humidified incubator with $5 \%$ $\mathrm{CO}_{2}$. All experiments used passage one fibroblasts from male and female fetuses.

\section{RNA extraction and amplification}

RNA was extracted using the TRIzol protocol (Invitrogen, Carlsbad, CA) according to the manufacturer's instructions. Subsequently, the MessageAmp antisense RNA (aRNA) kit (Ambion, Austin, TX) was used to amplify $1 \mu \mathrm{g}$ of extracted RNA from each group. To compare the different arrays between gestational time points, universal mouse RNA was amplified in individual reaction mixtures of $1 \mu \mathrm{g}$ aliquots simultaneously, with amplifications in the experimental sample and utilized as an internal amplification control. 


\section{Preparation of fluorescent complementary DNA probes}

Two micrograms of random hexamer and $4 \mu \mathrm{g}$ of RNA were heated at $65^{\circ} \mathrm{C}$ for $10 \mathrm{~min}$. Samples were then reverse transcribed with $2 \mathrm{mM}$ of each deoxyribose nucleotide triphosphate, $1 \times$ first-strand buffer, $0.5 \mu \mathrm{L}$ RNAse inhibitor, $200 \mathrm{U}$ superscript II, $10 \mathrm{mM}$ dithiothreitol, and either $3 \mu \mathrm{L}$ Cy3-deoxyribose uridine triphosphate (dUTP) (for experimental samples) or Cy5-dUTP (for universal mouse control samples) at $42^{\circ} \mathrm{C}$ for $1 \mathrm{~h}$ in a $30 \mu \mathrm{L}$ total reaction volume. To increase the reaction, $200 \mathrm{U}$ superscript II was added to the mixture and samples were incubated at $42^{\circ} \mathrm{C}$ for $1 \mathrm{~h}$. Fluorescent Cy3- or Cy5-labeled probes were washed with Tris-EDTA (TE) buffer $(10 \mathrm{mM}$ Tris, $1 \mathrm{mM}$ EDTA) through a microcon mini column (Millipore, Billerica, MA), treated with $450 \mu \mathrm{L} \mathrm{TE}$ buffer, and the inverted mini column was spun into a new tube. Microarray chips were immediately hybridized with probes.

\section{Pretreatment of microarray chips}

The Stanford Microarray Database Center was used to print microarray chips with 42,000 specific complementary DNAs (cDNAs) printed onto lysinecoated slides. Sequences and accession numbers can be accessed at http://genome-www5.stanford.edu// index.shtml, where single accession numbers from GenBank represent chosen cDNAs. Microarray chips were rehydrated before hybridization by being held down quickly over distilled water. Microarray chips were then quickly snap-dried over a $100^{\circ} \mathrm{C}$ heating block and DNA was crosslinked using a UV crosslinker $(300 \mathrm{~mJ})$.

\section{Microarray hybridization}

Fluorescent-labeled probes were heated at $100^{\circ} \mathrm{C}$ for $2 \mathrm{~min}$, denatured, and then incubated at $37^{\circ} \mathrm{C}$ for $20 \mathrm{~min}$. The recovered probe hybridization mixture with a volume of $32 \mu \mathrm{L}, 6.8 \mu \mathrm{L}$ of $20 \times$ saline sodium citrate (SSC), and $1.2 \mu \mathrm{L}$ of $10 \%$ sodium dodecyl sulfate (SDS) was placed onto microarray slides that were prewarmed. Coverslips were applied and slides were placed into a sealed moisture chamber at $65^{\circ} \mathrm{C}$ for $16 \mathrm{~h}$ to hybridize. Slides were then immediately washed with $1 \times \mathrm{SSC}$ in $0.03 \%$ SDS, with $0.5 \%$ SSC twice, and with $0.06 \%$ SSC twice. After the washes, slides were centrifuged at $84 \mathrm{~g}$ for $2 \mathrm{~min}$ and immediately scanned. An Axon microarray scanner (Molecular Devices, Sunnyvale, CA) was used for scanning slides.

\section{Microarray data analysis}

GenePix Pro 4.0 software (Molecular Devices) was used to analyze scanned slides. Densitometry data for gene identification and analysis were up- loaded into the Stanford Microarray Database. The log (base 2) of red/green normalized ratio (mean) was found and filtered based on a regression correlation of 0.6. Each gene was centered to the median and was only included in the final analysis if genes passed $>80 \%$ good data filter criterion. Pearson correlation was used to cluster genes. Subsequently, genes between E14 and E18 with significant differences were selected using significance analysis of microarrays (SAM). By utilizing a set of gene-specific $t$ tests, SAM is able to identify gene expression changes that are statistically significant. The analysis assigns a score to each individual gene dependent on the expression change based on the standard deviation of the gene repeated measurements. Permutations of repeated measurements are used to determine the false discovery rate (FDR) for genes equivalent to chance. Only genes that had both an FDR less than two and at least twofold expression difference were selected.

\section{Functional analysis of differentially expressed genes}

As previously described by Jovov et al., ${ }^{10}$ network and pathway analyses of probes were performed using Ingenuity Pathway Analysis (IPA; www.ingenuity.com, Ingenuity Systems, Redwood City, CA) between significantly regulated genes to identify functional connections. The significance of networks was calculated by IPA's integrated Ingenuity algorithm, which calculates $p$-values using the right-tailed Fisher's exact test for each canonical pathway. The association between a subset of genes from the whole experimental data set and a related function/pathway is evaluated to be due to random association.

\section{RESULTS \\ Differential gene expression between scarless E14 and scarring E18 fibroblasts}

SAM identified a total of 275 genes that were differentially expressed, with at least a twofold difference, in fetal fibroblasts between E14 and E18. Of these genes, 30 were significantly downregulated (Table 1) and 245 genes were significantly upregulated at the E18 compared with the E14 time point (Table 2).

\section{Functional pathway analysis}

Of the 245 genes upregulated in E18 compared with E14 fetal fibroblasts identified using microarray analysis (Fig. 1A), IPA identified 20 functional pathways represented by these genes (Fig. 1B). The top five pathways identified were associated with 
Table 1. Genes downregulated in E14 fibroblasts

\begin{tabular}{ll}
\hline Symbol & \multicolumn{1}{c}{ Gene Name } \\
\hline Arf4 & ADP-ribosylation factor 4 \\
Mfge8 & Milk fat globule-EGF factor 8 protein \\
Tmem147 & Transmembrane protein 147 \\
Mtg1 & Mitochondrial GTPase 1 homologue (Saccharomyces \\
& cerevisiae) \\
Galnt10 & UDP-N-acetyl-alpha-D-galactosamine:polypeptide \\
& N-acetylgalactosaminyltransferase 10 \\
Crmp1 & Collapsin response mediator protein 1 \\
Rpl27 & Ribosomal protein L27 \\
Timp3 & Tissue inhibitor of metalloproteinase 3 \\
1500003003Rik & RIKEN cDNA 1500003003 gene \\
Dpm1 & Dolichol-phosphate (beta-D) mannosyltransferase 1 \\
Csnk1a1 & Casein kinase 1, alpha 1 \\
Pgk1 & Phosphoglycerate kinase 1 \\
Pdcd6 & Programmed cell death 6 \\
Hdgf & Hepatoma-derived growth factor \\
Lama2 & Laminin, alpha 2 \\
Rab10 & RAB10, member RAS oncogene family \\
Tmem206 & Transmembrane protein 206 \\
Ubqln1 & Ubiquilin 1 \\
Myadm & Myeloid-associated differentiation marker \\
Mat2a & Methionine adenosyltransferase II, alpha \\
Parn & poly(A)-specific ribonuclease (deadenylation nuclease) \\
Rae1 & RAE1 RNA export 1 homologue (Schizosaccharomyces \\
Seh11 & pombe) \\
Rpl3 & SEH1-like (S. cerevisiae) \\
Sub1 & Ribosomal protein L3 \\
Nrbp2 & SUB1 homologue (S. cerevisiae) \\
Ppp3ca & Nuclear receptor binding protein 2 \\
Mtmr6 & Protein phosphatase 3, catalytic subunit, alpha isoform \\
Odc1 & Myotubularin-related protein 6 \\
Ttc12 & Ornithine decarboxylase, structural 1 \\
\hline & Tetratricopeptide repeat domain 12 \\
\hline &
\end{tabular}

colorectal cancer (CRC) metastasis signaling, hepatic fibrosis/hepatic stellate cell activation, pancreatic adenocarcinoma signaling, platelet-derived growth factor (PDGF) signaling, and endometrial cancer signaling. From the 30 genes downregulated in E18 compared with E14 fibroblasts, IPA identified 20 functional pathways represented by these genes (Fig. 1C). The five most significant functional pathways identified were putrescine biosynthesis III, s-adenosyl-l-methionine biosynthesis, d-myo-inositol $(1,4,5,6)$-tetrakisphosphate biosynthesis, d-myoinositol $(3,4,5,6)$-tetrakisphosphate biosynthesis, and dolichyl-diphosphooligosaccharide biosynthesis.

\section{DISCUSSION}

Previous work conducted by our laboratory analyzed the differences in the transcriptomes of fetal keratinocytes and fibroblasts between E16 and E18. At the time of this research, however, it was difficult to efficiently culture E14 fetal fibroblasts. ${ }^{11}$ Using a larger number of E14 fetal mice and a smaller culture plating surface in this study, we were able to successfully culture E14 fetal fibroblasts. The microarray analysis and signal pathway analysis conducted at the E14 time point in this study revealed a number of significant gene expression changes that occur during the transition period from scarless healing to healing with scarring. A recent investigation by our laboratory compared gene expression in fetal (E17) and adult mouse wounds and identified 178 genes upregulated and 13 genes downregulated in fetal compared with adult wounds. A selection of down- and upregulated pathways was identified (unpublished observations). Together with the findings presented here, this research contributes to unraveling the mechanism of fetal regenerative cutaneous wound repair. In the following sections, we characterize in greater detail some of the notable pathways differentially activated in E14 fibroblasts versus E18 fibroblasts.

\section{CRC metastasis signaling}

SAM revealed a significant increase in CRC metastasis signaling in E18 compared with E14 fetal fibroblasts. CRC tumorigenesis is well characterized and results from the accumulation of genetic mutations. Inflammation is a known environmental factor strongly associated with genetic mutations in cells of the colonic mucosa, which predisposes it to developing CRC. The association between CRC tumorigenesis and inflammation is demonstrated by the increased risk of CRC in patients with extensive and long-term colitis. ${ }^{12} \mathrm{~A}$ number of proinflammatory factors are activated by the network of CRC signaling, ${ }^{13}$ including $\mathrm{TNF} \alpha$, interleukin (IL)-8, IL-6, and VEGF, which are all found to be elevated in the serum of patients with CRC. ${ }^{14}$ Strong evidence identifies transforming growth factor- $\beta$ (TGF $\beta$ ) as one of the key factors promoting inflammation during tumorigenesis. ${ }^{15}$ Interestingly, inflammation and $\mathrm{TGF} \beta$ have also been demonstrated to play an immediate role in the fibrotic response to cutaneous injury, and TGF $\beta$ expression is significantly elevated in the fibroblasts present in keloid and hypertrophic scars. ${ }^{16} \mathrm{It}$ is highly likely, therefore, that the observed upregulation of CRC metastasis signaling in fetal fibroblasts at E18 compared with E14 is associated with increased levels of proinflammatory factors, including $\mathrm{TGF} \beta$, which ultimately contribute to scar formation. Consequently, targeted inhibition of one or more proinflammatory factors upregulated in scar-forming fetal fibroblasts could significantly improve the wound repair process in adult cutaneous wounds.

\section{Hepatic fibrosis/hepatic stellate cell activation}

A significant upregulation of hepatic fibrosis/ hepatic stellate cell activation pathway in fetal fibroblasts at E18 compared with E14 was found. 
Table 2. Genes upregulated in E14 fibroblasts

\begin{tabular}{|c|c|c|c|}
\hline Symbol & Gene Name & Symbol & Gene Name \\
\hline Lpar4 & Lysophosphatidic acid receptor 4 & Pex19 & Peroxisomal biogenesis factor 19 \\
\hline Ankrd17 & Ankyrin repeat domain 17 & Pikfyve & Phosphoinositide kinase, FYVE finger containing \\
\hline C330019G07Rik & RIKEN cDNA C330019G07 gene & Plac9 & Placenta specific 9 \\
\hline Cstf2t & Cleavage stimulation factor, $3^{\prime}$ pre-RNA subunit 2 , tau & Dstyk & Dual serine/threonine and tyrosine protein kinase \\
\hline Golt1b & Golgi transport 1 homologue B (S. cerevisiae) & Fcgbp & Fc fragment of $\lg G$ binding protein \\
\hline Adprh & ADP-ribosylarginine hydrolase & Pdgfa & Platelet-derived growth factor, alpha \\
\hline Ifrg15 & Interferon alpha responsive gene & Txndc16 & Thioredoxin domain containing 16 \\
\hline 4632428 N05Rik & RIKEN cDNA 4632428 N05 gene & Gatm & $\begin{array}{l}\text { Glycine amidinotransferase (L-arginine:glycine } \\
\text { amidinotransferase) }\end{array}$ \\
\hline Slc35a1 & Solute carrier family 35 (CMP-sialic acid transporter), member 1 & Foxn2 & Forkhead box N2 \\
\hline Gsk3b & Glycogen synthase kinase 3 beta & Mrpl49 & Mitochondrial ribosomal protein L49 \\
\hline Mepce & Methylphosphate capping enzyme & Rbm34 & RNA binding motif protein 34 \\
\hline Ficd & FIC domain containing & Bfar & Bifunctional apoptosis regulator \\
\hline Ptges & Prostaglandin E synthase & Mdh2 & Malate dehydrogenase 2, NAD (mitochondrial) \\
\hline BC016495 & cDNA sequence BC016495 & Wrnip1 & Werner helicase interacting protein 1 \\
\hline Myo10 & Myosin X & Thap7 & THAP domain containing 7 \\
\hline $\mathrm{Pbk}$ & PDZ binding kinase & Map1lc3b & Microtubule-associated protein 1 light chain 3 beta \\
\hline Tex19.1 & Testis expressed gene 19.1 & Hoxb2 & Homeobox B2 \\
\hline Mocs1 & Molybdenum cofactor synthesis 1 & Mphosph10 & $\begin{array}{l}\text { M-phase phosphoprotein } 10 \text { (U3 small nucleolar } \\
\text { ribonucleoprotein) }\end{array}$ \\
\hline Snap25 & Synaptosomal-associated protein 25 & Cyba & Cytochrome b-245, alpha polypeptide \\
\hline Adam10 & A disintegrin and metallopeptidase domain 10 & Slc9a6 & $\begin{array}{l}\text { Solute carrier family } 9 \text { (sodium/hydrogen exchanger), } \\
\text { member } 6\end{array}$ \\
\hline Idh3a & Isocitrate dehydrogenase $3(\mathrm{NAD}+)$ alpha & Pepd & Peptidase D \\
\hline Phtf2 & Putative homeodomain transcription factor 2 & Tmsb15l & Thymosin beta $15 \mathrm{~b}$ like \\
\hline Hist1 h2ae & Histone cluster 1, H2ae & Gadd $45 \mathrm{~g}$ & Growth arrest and DNA-damage-inducible 45 gamma \\
\hline Stmn3 & Stathmin-like 3 & Cct6a & Chaperonin containing Tcp1, subunit 6a (zeta) \\
\hline Ift74 & Intraflagellar transport 74 homologue (Chlamydomonas) & Smurf2 & SMAD-specific E3 ubiquitin protein ligase 2 \\
\hline Mett|21a & Methyltransferase-like 21A & Nol4 & Nucleolar protein 4 \\
\hline 112 & Interleukin 2 & Ppp1r2 & Protein phosphatase 1, regulatory (inhibitor) subunit 2 \\
\hline Ppp3cc & Protein phosphatase 3, catalytic subunit, gamma isoform & 4833439 L19Rik & RIKEN cDNA 4833439 L19 gene \\
\hline Ndufa13 & NADH dehydrogenase (ubiquinone) 1 alpha subcomplex, 13 & Tnfrsf12a & Tumor necrosis factor receptor superfamily, member 12a \\
\hline Gzmb & Granzyme B & Spire1 & Spire homologue 1 (Drosophila) \\
\hline Ccr5 & Chemokine (C-C motif) receptor 5 & Acadsb & Acyl-coenzyme A dehydrogenase, short/branched chain \\
\hline Mbd1 & Methyl-CpG binding domain protein 1 & Stat1 & Signal transducer and activator of transcription 1 \\
\hline Atp7a & ATPase, Cu++ transporting, alpha polypeptide & Notch4 & Notch gene homologue 4 (Drosophila) \\
\hline Psmc2 & Proteasome (prosome, macropain) 26S subunit, ATPase 2 & Cdc42ep5 & CDC42 effector protein (Rho GTPase binding) 5 \\
\hline Cenph & Centromere protein $\mathrm{H}$ & Corola & Coronin, actin binding protein $1 \mathrm{~A}$ \\
\hline Mllt3 & Translocated to, 3 & Map2k2 & Mitogen-activated protein kinase kinase 2 \\
\hline Zfp868 & Zinc finger protein 868 & Wnt4 & Wingless-related MMTV integration site 4 \\
\hline 4930528F23Rik & RIKEN cDNA 4930528F23 gene & Agpat3 & 1-acylglycerol-3-phosphate 0 -acyltransferase 3 \\
\hline Khdc1b & $\mathrm{KH}$ domain containing $1 \mathrm{~B}$ & Sh3d19 & SH3 domain protein D19 \\
\hline Tm4sf4 & Transmembrane 4 superfamily member 4 & Wdr54 & WD repeat domain 54 \\
\hline Mcm5 & $\begin{array}{l}\text { Minichromosome maintenance-deficient } 5 \text {, cell division cycle } 46 \\
\text { (S. cerevisiae) }\end{array}$ & Adal & Adenosine deaminase-like \\
\hline Fank1 & Fibronectin type 3 and ankyrin repeat domains 1 & Rpl19 & Ribosomal protein L19 \\
\hline Vegfc & Vascular endothelial growth factor C & Aasdhppt & $\begin{array}{l}\text { Aminoadipate-semialdehyde dehydrogenase- } \\
\text { phosphopantetheinyl transferase }\end{array}$ \\
\hline Parp16 & Poly (ADP-ribose) polymerase family, member 16 & Cdh3 & Cadherin 3 \\
\hline Ubxn8 & UBX domain protein 8 & Rsph9 & Radial spoke head 9 homologue (Chlamydomonas) \\
\hline DII1 & Delta-like 1 (Drosophila) & $\mathrm{HaO} 2$ & Hydroxyacid oxidase 2 \\
\hline Eef1b2 & Eukaryotic translation elongation factor 1 beta 2 & Syf2 & SYF2 homologue, RNA splicing factor (S. cerevisiae) \\
\hline Abhd14a & Abhydrolase domain containing $14 \mathrm{~A}$ & Pсp4l1 & Purkinje cell protein 4 -like 1 \\
\hline Ergic2 & ERGIC and golgi 2 & Appl1 & $\begin{array}{l}\text { Adaptor protein, phosphotyrosine interaction, } \mathrm{PH} \text { domain and } \\
\text { leucine zipper containing } 1\end{array}$ \\
\hline Josd2 & Josephin domain containing 2 & Cdk5rap3 & CDK5 regulatory subunit associated protein 3 \\
\hline Cyth1 & Cytohesin 1 & Tmem41b & Transmembrane protein $41 \mathrm{~B}$ \\
\hline Atp4a & ATPase, $\mathrm{H}+/ \mathrm{K}+$ exchanging, gastric, alpha polypeptide & G3bp2 & GTPase activating protein (SH3 domain) binding protein 2 \\
\hline Unkl & Unkempt-like (Drosophila) & Slc35b2 & Solute carrier family 35 , member B2 \\
\hline Ndufaf1 & $\begin{array}{l}\text { NADH dehydrogenase (ubiquinone) } 1 \text { alpha subcomplex, assembly } \\
\text { factor } 1\end{array}$ & Lmbrd1 & LMBR1 domain containing 1 \\
\hline Fam57a & Family with sequence similarity 57, member $A$ & Uckl1 & Uridine-cytidine kinase 1-like 1 \\
\hline Prkag1 & Protein kinase, AMP-activated, gamma 1 noncatalytic subunit & Scnm1 & Sodium channel modifier 1 \\
\hline
\end{tabular}


Table 2. (Continued)

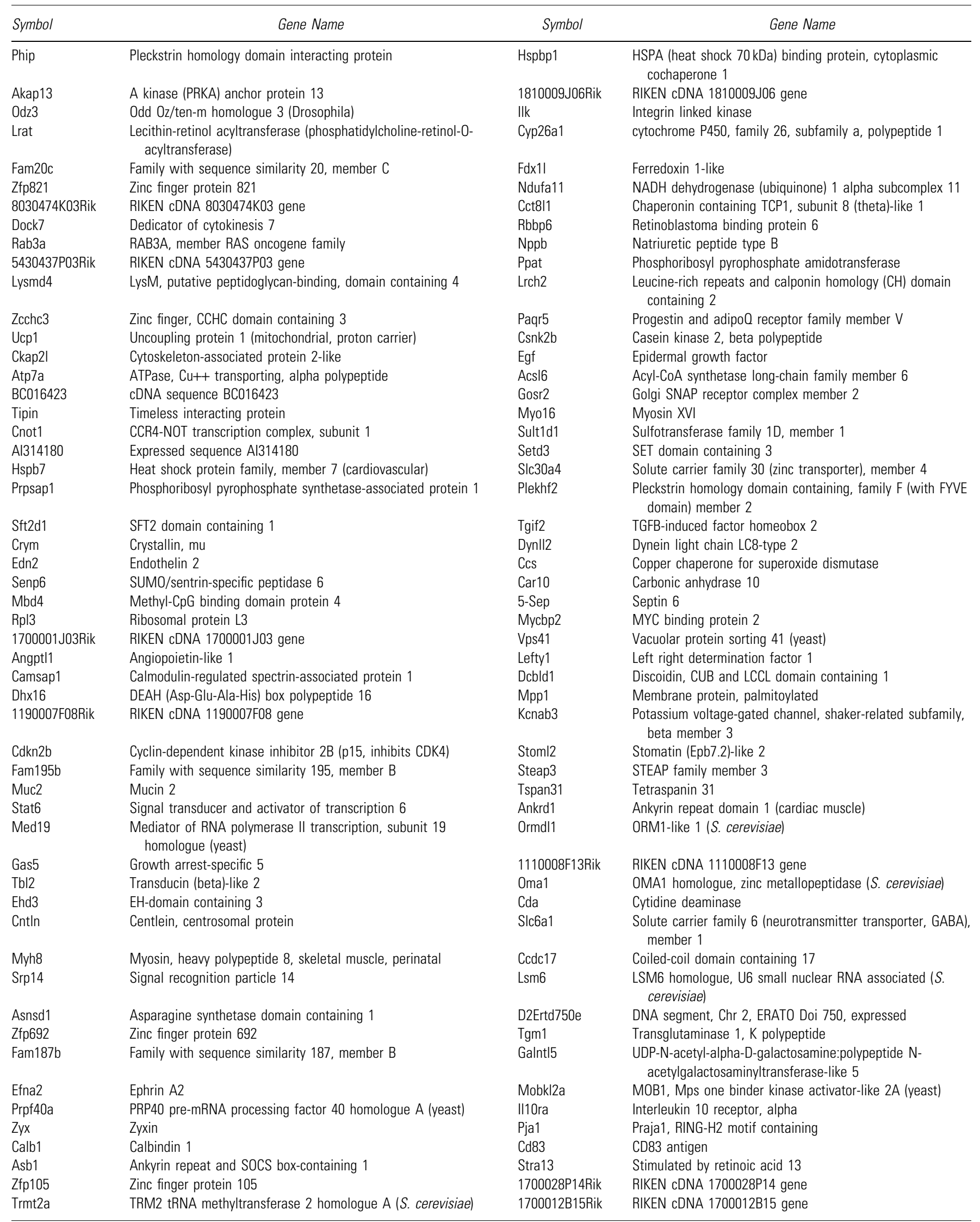


Table 2. (Continued)

\begin{tabular}{|c|c|c|c|}
\hline Symbol & Gene Name & Symbol & Gene Name \\
\hline Celf4 & CUGBP, Elav-like family member 4 & Chid1 & Chitinase domain containing 1 \\
\hline 1110034A24Rik & RIKEN cDNA 1110034 A24 gene & Chgb & Chromogranin B \\
\hline Acbd5 & Acyl-Coenzyme A binding domain containing 5 & Kctd14 & Potassium channel tetramerization domain containing 14 \\
\hline Gtf3c6 & General transcription factor IIIC, polypeptide 6, alpha & Rab11fip1 & RAB11 family interacting protein 1 (class I) \\
\hline Myc & Myelocytomatosis oncogene & Aldh3b1 & Aldehyde dehydrogenase 3 family, member B1 \\
\hline Rrp12 & Ribosomal RNA processing 12 homologue (S. cerevisiae) & Tpd52I1 & Tumor protein D52-like 1 \\
\hline Mapk10 & Mitogen-activated protein kinase 10 & 2210012G02Rik & RIKEN cDNA 2210012 G02 gene \\
\hline Pde6d & Phosphodiesterase 6D, cGMP-specific, rod, delta & Tspan3 & Tetraspanin 3 \\
\hline Mtus2 & Microtubule-associated tumor suppressor candidate 2 & Rbm39 & RNA binding motif protein 39 \\
\hline Pon1 & Paraoxonase 1 & Bin1 & Bridging integrator 1 \\
\hline Sfxn1 & Sideroflexin 1 & Smc3 & Structural maintenance of chromosomes 3 \\
\hline Pak2 & p21 protein (Cdc42/Rac)-activated kinase 2 & Dnttip1 & Deoxynucleotidyltransferase, terminal, interacting protein 1 \\
\hline Etv6 & ETS variant gene 6 (TEL oncogene) & Dcaf4 & DDB1- and CUL4-associated factor 4 \\
\hline
\end{tabular}

This pathway involves activation of the hepatic stellate cell following liver injury, the principal effector of hepatic fibrogenesis, which becomes highly proliferative and synthesizes a fibrotic matrix that is rich in type I collagen. ${ }^{17}$ Hepatic fibrosis can arise secondary to a number of factors causing liver injury, including viral hepatitis or alcohol abuse. Regardless of the precise etiology, hepatic fibrosis is ultimately characterized by an increase in ECM deposition and formation of a hepatic scar. Immediately following liver injury, there is a marked transition of hepatic stellate cells from a quiescent to a robust, activated state. This response is elicited by neighboring Kupffer, endothelial, and injured hepatocyte cells, which release potent reactive oxygen intermediates (ROI) that exert paracrine stimulation of stellate cells. ${ }^{18}$ Excessive accumulation of reactive oxygen species (ROS) in wounds significantly impairs wound healing and causes substantial tissue damage. ${ }^{19}$ Our findings here suggest a differential stellate cell activation pathway between the E14 and E18 time points in fetal fibroblasts, and this could be partially caused by increased ROS in the wound bed at E18. Accordingly, methods that eliminate accumulated ROS in wound beds may enhance the regenerative capability of skin.

In addition to activating stellate cells, endothelial cells also activate plasmin. Plasmin stimulates the conversion of latent TGF $\beta 1$ to an active fibrogenic form. ${ }^{20}$ TGF $\beta$, as mentioned, plays a major role in the fibrotic response to injury and the formation of scars. Therefore, targeted inhibition of the conversion of TGF $\beta 1$ into its more active fibrogenic form will likely enhance the regenerative properties of skin once beyond the E14 time point.

\section{PDGF signaling}

Our microarray analysis demonstrated significantly increased expression of PDGF in E18 com- pared with E14 fetal fibroblasts. Higher levels of PDGF have been documented both in adult wounds compared with embryonic wounds, and in uninjured adult skin compared with uninjured embryonic skin. ${ }^{21,22}$ No previous work has specifically compared the differential activation of PDGF signaling between early scar-free fetal skin and later scar-forming fetal skin. The functional pathway analysis here suggests that increased PDGF signaling contributes to the loss of the regenerative healing capability between the E14 and E18 time points. This finding is further supported by the role of PDGF in mediating proliferation and differentiation of fibroblasts. PDGF expression also leads to subsequent upregulation of TGF $\beta 1$ receptors, which have a major role in the fibrotic response to tissue injury.

\section{Putrescine biosynthesis III}

Microarray analyses showed greater downregulation of putrescine biosynthesis in E18 compared with E14 fetal fibroblasts. Putrescine has been identified to play a significant role in cell proliferation in response to injury, and is required for the completion of DNA synthesis. ${ }^{23}$ Putrescine is also suggested to be an integral precursor for the development of complex polyamines, which are major regulatory factors of mammalian tissues and influence both growth and signal transduction. ${ }^{24}$ The formation of new tissue, through cellular proliferation and migration of cells to the wound site, is a key part of wound healing. Downregulation of the putrescine biosynthesis III pathway at E18 may impair signaling, impact the regulation of cellular proliferation, and ultimately increase the amount of new tissue formed, leading to the formation of a scar. Further exploration of the putrescine biosynthesis III pathway could reveal a target for drug therapy that could potentially rescue regenerative capability at scar-forming time points. 

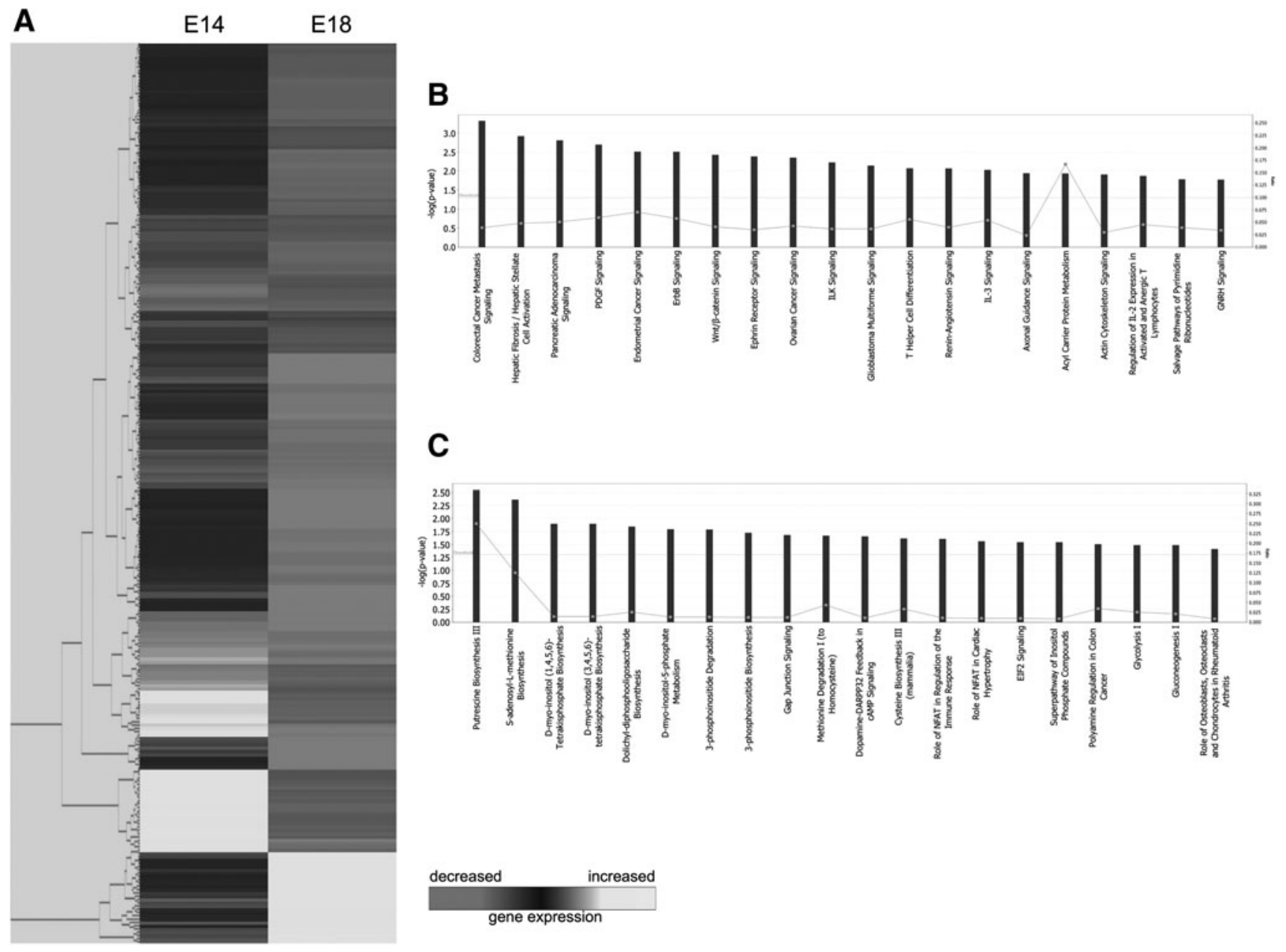

Figure 1. Microarray analysis of E14 and E18 fibroblasts. (A) Hierarchical clustering of upregulated and downregulated genes from fetal fibroblasts at E14 versus E18. Individual genes cluster according to the dendrogram on the left and are visually represented in the heat map on the right, blue indicates downregulation and yellow indicates upregulation. (B) Canonical pathways identified via IPA that were significantly enriched for among genes observed to be substantially upregulated. (C) Canonical pathways identified via IPA that were significantly enriched for among genes observed to be substantially downregulated. E14, embryonic day 14; E18, embryonic day 18; IPA, Ingenuity Pathway Analysis.

\section{D-myo-inositol $(3,4,5,6)$-tetrakisphosphate biosynthesis}

Microarray analysis revealed that d-myo-inositol $(3,4,5,6)$-tetrakisphosphate biosynthesis was downregulated in E18 compared with E14 fetal fibroblasts. D-myo-inositol $(3,4,5,6)$-tetrakisphosphate is part of the inositol phosphate family, which are intracellular signaling molecules regulating the crucial functions of cell growth, apoptosis, and cell differentiation. Previous work has tentatively identified the target of d-myo-inositol $(3,4,5,6)$ tetrakisphosphate as a plasma membrane $\mathrm{Ca}^{2+}$ activated chloride channel. ${ }^{25}$ Interestingly, blockage of chloride channels severely reduces the rate of wound healing. ${ }^{26}$ Downregulation of chloride channel function at E18 may reduce healing capability in fetal wounds and prolong the formation of new tissue and scars. D-myo-inositol $(3,4,5,6)$ tetrakisphosphate is also involved in mediating the ability of remodeling complexes to induce transcription in phosphate-responsive genes. ${ }^{27}$ This finding suggests that reduction in d-myo-inositol $(3,4,5,6)$-tetrakisphosphate activity may contribute to epigenetic silencing and consequently the loss of regenerative healing capability observed at E18. Further work with d-myo-inositol $(3,4,5,6)$ tetrakisphosphate biosynthesis could reveal a novel target to induce regenerative healing mechanisms.

\section{INNOVATION}

Previous work has identified the key time point during fetal development when the ability to heal without scar is lost. However, the mechanism driving the transition from scarless, regenerative fetal wound healing to scar-forming adult wound repair is still largely unknown. Our work significantly narrows the search to a set of key genes and 


\section{KEY FINDINGS}

- Thirty genes were significantly downregulated in E18 fetal fibroblasts compared with E14 fetal fibroblasts.

- Enrichment analysis revealed over 20 pathways were downregulated in E18 fetal fibroblasts compared with E14 fetal fibroblasts, including putrescine biosynthesis III, s-adenosyl-I-methionine biosynthesis, d-myoinositol $(1,4,5,6)$-tetrakisphosphate biosynthesis, d-myo-inositol $(3,4,5,6)$ tetrakisphosphate biosynthesis, and dolichyl-diphosphooligosaccharide biosynthesis.

- Two hundred forty-five genes were significantly upregulated in E18 fetal fibroblasts compared with E14 fetal fibroblasts.

- Enrichment analysis revealed over 20 pathways upregulated in E18 fetal fibroblasts compared with E14 fetal fibroblasts, including CRC metastasis signaling, hepatic fibrosis/hepatic stellate cell activation, pancreatic adenocarcinoma signaling, PDGF signaling, and endometrial cancer signaling.

\section{AUTHOR DISCLOSURE AND GHOSTWRITING}

No competing financial interests exist. The content of this article was expressly written by the authors listed. No ghostwriters were used to write this article.

\section{ABOUT THE AUTHORS}

Michael S. Hu, MD, MPH, MS, is a postdoctoral fellow at Stanford interested in stem cell biology and regenerative medicine. $\mathrm{He}$ is pursuing a career in craniofacial plastic surgery. Mimi R. Borrelli, MBBS, MSs, BSc, is a postdoctoral scholar at Stanford. Michael Januszyk, $\mathbf{M D}, \mathbf{P h D}$, is a resident in plastic surgery at UCLA. Anna Luan, MD, MS, pathways that drive the scar-free healing mechanism. Further detailed examination of these genes and signaling pathways will give us a better understanding of the mechanism behind scarless wound healing and potentially identify targets for future therapies.

\section{ACKNOWLEDGMENTS AND FUNDING SOURCES}

This work was supported, in part, by an NIH grant R01 GM087609 (to H.P.L.), a gift from Ingrid Lai and Bill Shu in honor of Anthony Shu (to H.P.L.), and the Hagey Laboratory for Pediatric Regenerative Medicine and Children's Surgical Research Program (to M.T.L. and H.P.L.). Additional funding was provided by the American Society of Maxillofacial Surgeons (ASMS)/Maxillofacial Surgeons Foundation (MSF) Research Grant Award (to M.S.H., M.T.L., and H.P.L.), the Sarnoff Cardiovascular Research Foundation (to W.X.H.), the California Institute for Regenerative Medicine (CIRM) Clinical Fellow training grant TG2-01159 (to M.S.H.), and the Stanford University School of Medicine Transplant and Tissue Engineering Fellowship Award (to M.S.H.). is a resident in plastic surgery at Stanford. Samir Malhotra, BS, is a premedical undergraduate student at Stanford. Graham G. Walmsley, MD, $\mathbf{P h D}$, is a stem cell scientist working in venture capital. Wan Xing Hong, MD, is a resident in general surgery at Stanford. Ruth Terlin, MB, BCh, BAO, MRCSI, MD, is a resident in plastic surgery at Stanford. Geoffrey C. Gurtner, MD, is a Professor of Surgery and Vice Chairman for Research in the Department of Surgery at Stanford. Michael T. Longaker, MD, MBA, is a Professor of Surgery and Bioengineering at Stanford. He is the Director of Research for the Program in Regenerative Medicine, Children's Surgical Research, and Division of Plastic and Reconstructive Surgery. His extensive research experience includes wound stem cell biology. H. Peter Lorenz, MD, is a Professor and Chief of Plastic Surgery at the Lucile Packard Children's Hospital at Stanford. His clinical interests are in craniofacial surgery, pediatric plastic surgery, and reconstructive and cosmetic surgery. His laboratory group is studying mechanisms underlying scarless skin healing and the function of progenitor cells during wound repair/regeneration. healing, tissue engineering, and developmental/

\section{REFERENCES}

1. Larson BJ, Longaker MT, Lorenz HP. Scarless fetal wound healing: a basic science review. Plast Reconstr Surg 2010;126:1172.

2. Rowlatt U. Intrauterine wound healing in a 20 week human fetus. Virchows Arch 1979;381:353-361.

3. Adzick NS, Longaker MT. Scarless fetal healing Therapeutic implications. Ann Surg 1992;215:3.
4. Cass DL, Bullard KM, Sylvester KG, Yang EY, Longaker MT, Adzick NS. Wound size and gestational age modulate scar formation in fetal wound repair. J Pediatr Surg 1997;32:411-415.

5. Colwell AS, Krummel TM, Longaker MT, Lorenz HP. An in vivo mouse excisional wound model of scarless healing. Plast Reconstr Surg 2006;117 2292-2296.
6. Lo DD, Zimmermann AS, Nauta A, Longaker MT, Lorenz HP. Scarless fetal skin wound healing update. Birth Defects Res C Embryo Today 2012; 96:237-247.

7. Brown B, McKenna S, Siddhi K, McGrouther D, Bayat $A$. The hidden cost of skin scars: quality of life after skin scarring. J Plast Reconstr Aesthet Surg 2008;61:1049-1058. 
8. Alster TS, Tanzi EL. Hypertrophic scars and keloids. Am J Clin Dermatol 2003;4:235-243.

9. Gurtner GC, Werner S, Barrandon Y, Longaker MT. Wound repair and regeneration. Nature 2008;453: 314-321.

10. Jovov B, Araujo-Perez F, Sigel CS, et al. Differential gene expression between African American and European American colorectal cancer patients. PLoS One 2012;7:e30168.

11. Hu MS, Januszyk M, Hong WX, et al. Gene expression in fetal murine keratinocytes and fibroblasts. J Surg Res 2014;190:344-357.

12. Itzkowitz SH, Yio X. Inflammation and cancer IV. Colorectal cancer in inflammatory bowel disease: the role of inflammation. Am J Physiol Gastrointest Liver Physiol 2004;287:G7-G17.

13. Coussens LM, Werb Z. Inflammation and cancer. Nature 2002;420:860

14. Klampfer L. Cytokines, inflammation and colon cancer. Curr Cancer Drug Targets 2011;11:451-464

15. Bierie B, Moses HL. Transforming growth factor beta (TGF- $\beta$ ) and inflammation in cancer. Cytokine Growth Factor Rev 2010;21:49-59.

16. Leask A, Abraham DJ. TGF- $\beta$ signaling and the fibrotic response. FASEB J 2004;18:816-827.

17. Reeves HL, Friedman SL. Activation of hepatic stellate cells-a key issue in liver fibrosis. Front Biosci 2002;7:d808-d826.
18. Friedman SL. The cellular basis of hepatic fibrosis-mechanisms and treatment strategies. $\mathrm{N}$ Engl J Med 1993;328:1828-1835.

19. Rasik AM, Shukla A. Antioxidant status in delayed healing type of wounds. Int J Exp Pathol 2000;81: 257-263.

20. Friedman SL. Cytokines and fibrogenesis. In: Seminars in liver disease. Semin Liver Dis 1999;2: 129-140.

21. Ferguson MW, O'Kane S. Scar-free healing: from embryonic mechanisms to adult therapeutic intervention. Philos Trans R Soc Lond B Biol Sci 2004:359:839-850.

22. Song $H$, Chai J, Lin Z, et al. A comparative study of PDGF and EGF expression in skin wound healing between human fetal and adult. Zhonghua Zheng Xing Wai Ke Za Zhi 2003;19:199-202.

23. Sunkara PS, Rao PN, Nishioka K. Putrescine biosynthesis in mammalial cells: essential for DNA synthesis but not for mitosis. Biochem Biophys Res Commun 1977;74:1125-1133.

24. Coleman CS, Guirong H. Putrescine biosynthesis in mammalian tissues. Biochem J 2004;379:849-855.

25. Ismailov II, Fuller CM, Berdiev BK, Shlyonsky VG, Benos DJ, Barrett KE. A biologic function for an "orphan" messenger: D-myo-inositol 3, 4, 5, 6tetrakisphosphate selectively blocks epithelial calcium-activated chloride channels. Proc Natl Acad Sci U S A 1996;93:10505-10509.
26. Fuchigami T, Matsuzaki T, Ihara S. Possible roles of $\mathrm{ENaC}$ and $\mathrm{Cl}-$ channel in wound closure in Xenopus laevis embryos. Zoolog Sci 2011;28:703-711.

27. Steger DJ, Haswell ES, Miller AL, Wente SR, O'shea EK. Regulation of chromatin remodeling by inositol polyphosphates. Science 2003;299:114116

\section{Abbreviations and Acronyms}

aRNA = antisense RNA

$\mathrm{cDNA}=$ complementary DNA

$\mathrm{CRC}=$ colorectal cancer

dUTP $=$ deoxyribose uridine triphosphate

$\mathrm{E} 14=$ embryonic day 14

$\mathrm{E} 18=$ embryonic day 18

$\mathrm{ECM}=$ extracellular matrix

EDTA = ethylenediaminetetraacetic acid

$\mathrm{FDR}=$ false discovery rate

$\mathrm{IL}=$ interleukin

IPA = Ingenuity Pathway Analysis

$\mathrm{NIH}=$ National Institutes of Health

PDGF = platelet-derived growth factor

$\mathrm{ROI}=$ reactive oxygen intermediates

ROS $=$ reactive oxygen species

SAM = significance analysis of microarrays

SDS $=$ sodium dodecyl sulfate

SSC $=$ saline sodium citrate

$\mathrm{TE}=$ Tris-EDTA

TGF $\beta=$ transforming growth factor $-\beta$ 